\title{
Coherent structures and chaotic advection in three dimensions
}

\author{
STEPHEN WIGGINS
}

Department of Mathematics, University of Bristol, Bristol BS8 1TW, UK

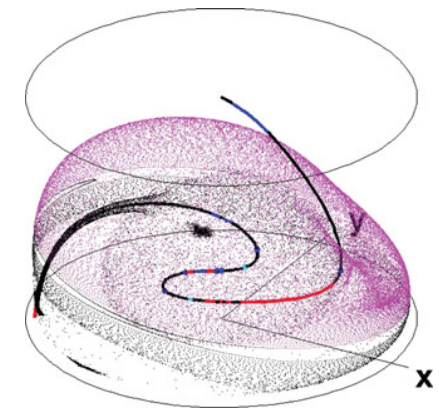

In the 1980 s the incorporation of ideas from dynamical systems theory into theoretical fluid mechanics, reinforced by elegant experiments, fundamentally changed the way in which we view and analyse Lagrangian transport. The majority of work along these lines was restricted to two-dimensional flows and the generalization of the dynamical systems point of view to fully three-dimensional flows has seen less progress. This situation may now change with the work of Pouransari et al. (J. Fluid Mech., this issue, vol. 654, 2010, pp. 5-34) who study transport in a three-dimensional time-periodic flow and show that completely new types of dynamical systems structures and consequently, coherent structures, form a geometrical template governing transport.

Key words: chaotic advection, coherent structures, dynamical systems, transport

\section{Introduction}

The 1980s saw the rise in applied dynamical systems theory. A consequence of this was the creation of a large amount of phenomenology, often guided by existing theory, related to two-dimensional area preserving maps. At the same time the widespread recognition that the motion of fluid particles in two-dimensional incompressible timeperiodic flow was equivalent to an area preserving map meant that the variety of phase space structures found in two-dimensional area preserving maps might offer new insights into fluid transport and mixing (Aref 1984). The development that solidified the merging of these two areas was a series of elegant experiments that illustrated the role of 'dynamical systems structures' in Lagrangian transport and mixing.

A compelling aspect for the use of the dynamical systems approach to Lagrangian transport was that it provided a theoretical and computational setting leading to the realization and exploitation of earlier physical insights. A notable example was the stretching and folding picture of mixing due to Reynolds, described in detail in Ottino, Jana \& Chakravarthy (1994), which received no attention until the 1980s. In particular, 'stretching and folding' was quantified through the idea of a Smale horseshoe map, which characterized regions of the flow that stretch and fold in a particular manner, giving rise to a mathematically precise form of deterministic chaos. In a seminal experiment, the existence of horseshoes, and the role they play in mixing, was demonstrated in Chien, Rising \& Ottino (1986). Examining the 'horseshoe mechanism' more deeply, lower dimensional 'structures' - the stable and unstable manifolds of hyperbolic (saddle-like) fluid particle trajectories - formed 
the geometrical template in the flow responsible for circumstances leading to the horseshoe map. This is the content of the Smale-Birkhoff homoclinic theorem. Further analysis of this geometrical template led to the development of lobe dynamics, which describes how regions of fluid move throughout chaotic regions (a theory developed in Rom-Kedar, Leonard \& Wiggins 1990, with experimental confirmation in Horner et al. 2002).

A complementary approach is through the consideration of 'non-mixing', i.e. the nature of regions of 'trapped' fluid that cannot mix with fluid outside the region. These are referred to as 'islands', and dynamical systems theory gives them a precise meaning through the Kolmogorov-Arnold-Moser (KAM) theorem, beautifully visualized in Kusch \& Ottino (1992). Most significantly, the KAM theorem also predicts that they exist 'sufficiently close to', and surrounding, elliptic (linearly stable) periodic fluid particle trajectories. A common theme is that certain types of flow structures allow us to infer the nature of transport and mixing of specific regions of the flow. These flow structures are all examples of invariant manifolds (dynamical systems terminology) or material curves (fluid mechanical terminology). Identifying and characterizing invariant manifolds is a topic of central interest in dynamical systems theory, and, as we have described above, the techniques that have been developed have an immediate implication for mixing and transport in fluids. Invariant manifolds are the rigorous mathematical manifestation of the fluid mechanical notion of coherent structures, allowing for description, prediction and computation.

\section{Overview}

Essentially all of the work described above is for two-dimensional incompressible time-periodic flows, and of the little work that has been done in three dimensions, most involves the case of a spatial periodicity which reduces it to the two-dimensional time-periodic case. The natural question is 'how does the dynamical systems approach generalize to three dimensions?' Can horseshoe maps be constructed and have the same implications for mixing and transport? Is there an analogue to an island in three dimensions? Is there a lobe dynamics that describes the motion of fluid through the complex geometrical template formed by the intersecting stable and unstable manifolds? These notions do generalize to three dimensions, but their implications for fluid transport and mixing are, in general, very different. More significantly, however, is that there are completely new geometrical structures - invariant manifolds - governing new transport and mixing mechanisms in three dimensions.

This is most easily seen by asking the question 'what is an island'? In the twodimensional case we could use the KAM theorem to conclude that KAM tori surrounded elliptic periodic points of the associated Poincare map. These resulted in 'trapped' regions of fluid that did not mix with their surroundings. The standard KAM theorem does not apply to odd dimensional dynamical systems, but generalizations of the KAM theorem have been developed that allow one to conclude that under certain conditions two-dimensional KAM tori exist in the Poincare maps associated with three-dimensional time-periodic flows (Cheng \& Sun 1990). Most significantly, the 'center' of such a KAM tube is a (normally) elliptic periodic invariant curve. This is a structure unique to three dimensions and while the theory has not been fully developed, some spectacular visualizations of 'three-dimensional islands' can be seen in Fountain, Khakhar \& Ottino (1998). Normally hyperbolic periodic invariant curves having two-dimensional stable and unstable manifolds also exist, and from these manifolds a lobe dynamics can be developed. Moreover, the intersection of 
these stable and unstable manifolds provides a new mechanism for chaos in three dimensions.

While fragments of the mathematics necessary to realize this 'three-dimensional picture' have been developed over the past 15 years (Mezic \& Wiggins 1994), they have not been embraced by fluid mechanicians. There are, probably, several reasons for this. One is that it is more difficult to visualize, both mentally and computationally, geometrical objects in three dimensions. Another is that the mathematics is, relatively, new, and not widely known. But probably the most important reason is that there has not been a compelling example showing that these three-dimensional dynamical systems tools are necessary for describing fluid transport. This has changed with the recent work of Pouransari, Speetjens \& Clercx (2010).

They consider a three-dimensional lid-driven cylinder flow where the fluid is set in motion through periodic forcing resulting from in-plane motion of the lower endwall. Three forcing protocols are considered. They first consider the non-inertial limit - zero Reynolds number $(R e=0)$. A significant issue in three dimensions is the determination of invariant manifolds in the flow. For two-dimensional flows the 'seeds' of coherent structures are periodic particle trajectories, which may still play a role, but in three dimensions there is the possibility of periodic invariant curves and two-dimensional invariant surfaces. Pouransari et al. (2010) use a powerful method based on symmetries that they developed in Speetjens, Clercx \& van Heijst (2004) to identify periodic invariant curves. They then determine the stability of these curves and, in general, find that a particular curve may be normally hyperbolic along a certain length but change to normally elliptic along the rest of the length. The point where the curve changes stability is normally parabolic, and this 'exceptional' point plays an important role for non-zero $R e$. They also discuss two-dimensional invariant surfaces given by constants of the motion and show that the flow structure is such that it is 'filled out' by concentric, invariant spheres. The particle motion on the invariant spheres can take a variety of forms, depending on the radius of the sphere, as well as the forcing. In particular, invariant spheres can exhibit particle motions that appear integrable (regular), a mixture of regular and chaotic, and completely chaotic. Moreover, the dynamics of particle motion on the sphere is governed by two-dimensional time-periodic Hamiltonian dynamics.

For $R e=0$ the particle motion is not three-dimensional, being confined to twodimensional invariant spheres. Nevertheless, understanding the invariant manifold structure for $R e=0$ is a crucial step for understanding fully three-dimensional motion for small, but non-zero Reynolds number since the invariant manifold structure for $R e=0$ will provide the 'skeleton' governing fully three-dimensional transport. A central question is whether or not the invariant spheres are destroyed for non-zero Reynolds number. Pouransari et al. (2010) find the intriguing result that those on which the particle motion appears to be fully chaotic persist for 'long times'. This is reminiscent of KAM-type results where tori on which the dynamics is ergodic persist under perturbation. This is significant since the persistence of even one invariant sphere would be sufficient to prohibit transport throughout the entire domain. They also consider the behaviour of the periodic invariant curves for non-zero Reynolds number. An important observation is the role that they play in causing the 'breakdown' of the invariant spheres. This can occur when a periodic invariant curve intersects an invariant sphere in a parabolic point. Parabolic points typically undergo bifurcation under perturbation, and this can result in a 'hole' forming in the invariant sphere allowing for transport through the sphere. This phenomenon is called 'resonanceinduced merger' (RIM) by Pouransari et al. (2010). Moreover, 'KAM-like tori', or 
tubes, appear to surround the normally elliptic segments of the invariant curves, and this can result in a complex geometric structure involving tubes connecting spheres. They show that the complexity of this arrangement depends on the forcing protocol. RIM appears to play a central 'organizing' role in each case, and it may well be a 'universal' mechanism underlying the formation of such coherent structures in three-dimensional time-dependent flows. Litvak-Hinenzon \& Rom-Kedar (2002) and Vainchtein, Neishtadt \& Mezic (2006) may well be relevant.

\section{Future}

The work of Pouransari et al. (2010) has the potential to stimulate many avenues of research on Lagrangian transport and mixing in three dimensions. Experiments played a fundamental role in establishing the dynamical systems point as essential for our understanding of mixing and transport in two-dimensional time-periodic flows. The same will be true for three-dimensions and Pouransari et al. (2010) have provided a 'roadmap' for experimental investigations. Such experiments have become feasible recently due to advancements such as three-dimensional 'particle tracking velocimetry' and 'laser-induced fluorescence' (for concentration fields).

\section{References}

Aref, H. 1984 Stirring by chaotic advection. J. Fluid Mech. 143, 1-21.

ChEng, C.-G. \& SuN, Y.-S. 1990 Existence of invariant tori in three-dimensional measure preserving mappings. Cel. Mech. 47, 275-292.

Chien, W. L., Rising, H. \& Ottino, J. M. 1986 Laminar and chaotic mixing in several cavity flows. J. Fluid. Mech. 170, 355-377.

Fountain, G. O., KhakhaR, D. V. \& Ottino, J. M. 1998 Visualization of three-dimensional chaos. Sci. 281, 683-686.

Horner, M., Metcalfe, G., Wiggins, S. \& Ottino, J. M. 2002 Transport enhancement mechanisms in open cavities. J. Fluid Mech. 452, 199-229.

Kusch, H. A. \& Ottino, J. M. 1992 Experiments on mixing in continuous chaotic flows. J. Fluid Mech. 236, 319-348.

Litvak-Hinenzon, A. \& Rom-Kedar, V. 2002 Parabolic resonance in 3 degree-of-freedom near integrable Hamiltonian systems. Physica D 164 (3-4), 213-250.

Mezic, I. \& Wiggins, S. 1994 On the integrability and perturbation of three-dimensional fluid flows with symmetry. J. Nonlinear Sci. 4, 157-194.

Ottino, J. M., Jana, S. C. \& Chakravarthy, V. S. 1994 From Reynolds's stretching and folding to mixing studies using horseshoe maps. Phys. Fluids 6 (2), 685-699.

Pouransari, Z., Speetjens, M. F. M. \& Clercx, H. J. H. 2010 Formation of coherent structures by fluid inertia in three-dimensional laminar flows. J. Fluid Mech. 654, 5-34.

Rom-Kedar, V., Leonard, A. \& Wiggins, S. 1990 An analytical study of transport, mixing, and chaos in an unsteady vortical flow. J. Fluid Mech. 214, 347-394.

Speetjens, M. F. M., Clercx, H. J. H. \& van Heijst, G. J. F. 2004 A numerical and experimental study on advection in three-dimensional Stokes flow. J. Fluid Mech. 514, 77-105.

Vainchtein, D., Neishtadt, A. \& Mezic, I. 2006 On passage through resonances in volumepreserving systems. Chaos 16, 043123. 